\title{
Editorial
}

Elisabeth Sorantin*

\section{CEEPUS: Active Methods in Setting up a New Regional Academic Exchange Program}

https://doi.org/10.1515/edu-2020-0132

received August 11, 2020; accepted December 5, 2020.

\begin{abstract}
CEEPUS - Central European Exchange Program for University Studies - is the biggest regional academic exchange program in Europe, currently comprising 15 countries. CEEPUS awards mobility grants to students and teachers, mostly in the framework of networks. From a much-doubted bold experiment, CEEPUS has evolved into a best practice example and has more than fulfilled its original expectations. As CEEPUS III is to expire on April 30,2025 , the discussion is on whether and in which form a possible CEEPUS IV shall be developed.
\end{abstract}

Keywords: academic exchange program; CEEPUS.

\section{Introduction}

The fall of the Iron Curtain brought new challenges for academic communities in Central and Eastern Europe. At a ministerial meeting in Graz in late 1992, regional ministers expressed their concern that there was no suitable framework for multilateral academic cooperation for universities from the former East Bloc. Another reason for concern was that except for Austria none of the Central European countries was entitled to participate in the new Erasmus program. It was feared that once these countries could participate, their universities would lack the necessary skills to do so successfully.

The only way to handle these challenges was the bold decision to devise a new regional multilateral exchange program among equal partners: CEEPUS.

\section{Implementation}

\subsection{Facts and figures}

- Legal framework: International Agreement, currently CEEPUS III.

- Founding countries: Austria, Bulgaria, Hungary, Poland, Slovak Republic, Slovenia.

- Member states 2020: Albania, Austria, Bosnia and Herzegovina, Bulgaria, Croatia, the Czech Republic, Hungary, North Macedonia, Moldova, Montenegro, Poland, Romania, Serbia, the Slovak Republic and Slovenia. Prishtina et al. are also participating.

\section{Important players:}

- Joint Committee of Ministers: Takes all important decisions, pledges scholarship months each academic year. CEEPUS III (2010), Art 3.

Working Groups of the Joint Committee of Ministers: IC - International Commission: In charge of network selection, made up of experts and National CEEPUS Offices. Chaired by the Central CEEPUS Office, which is neutral.

SOM - Senior Officials' Meeting: Senior Officials in charge of CEEPUS at the respective ministry, address current issues and pass recommendations to the Joint Committee of Ministers. Chaired by the Central CEEPUS Office, which is neutral.

- Central CEEPUS Office: Coordinates the whole program, makes proposals for further program development. Staff: 1 fulltime, 2 part time. CEEPUS III (2010), Art 5.

- National CEEPUS Offices: In charge of national implementation. Staff: $1-2$ officers, not in all cases full time. CEEPUS III (2010), Art 4.

- No. of awarded scholarship months since 1995 until spring 2020: approx. 75.000 .

*Corresponding author: Elisabeth Sorantin, CEEPUS Secretary General 1995 - 2020, Austria, E-mail: e.sorantin@gmail.com 


\subsection{Objectives and main features The new program was to be as user friendly as possible. To ensure easy access, par- ticipation was foreseen on department or faculty level, not on university level.}

Focusing on the formation of networks seemed the best approach to connect players in the field across the region. Basically the only entry ticket for participation was that one's institution was on the list of eligible institutions as defined by each member state annually (CEEPUS III, 2010, Art 2, para 1) and that a Letter of Endorsement guaranteeing that CEEPUS students were to be exempt from tuition signed by the rector/vice rector was submitted. Mutual recognition of CEEPUS courses and lectures had to be guaranteed as well.

Size matters: A network had to consist of at least three participating units from different countries.

As for financing, there was to be no transfer of funds. Instead, an internal currency was devised, "1 scholarship month". In order to allow for a critical mass of scholarship months, each country was obliged to pledge at least 100 months per academic year. Scholarship months were understood as comprehensive grants according to the local standard of living and were to be safeguarded against inflation. Grants were to be awarded to applicants exchanged within networks or to Freemovers.

Finally, the new program was to be very affordable for the member states financing it, i.e. to operate with a minimum of staff and be based on lean management.

\subsection{Important aspects of practical implementation}

CEEPUS as a bold new idea also needed a bold new administrative approach to implement its vision.

The CEEPUS corporate credo could be summed up like this:

- Devise a flexible legal framework.

- Keep administration as simple and user-friendly as possible.

- Consider first what your clients need and scale down administrative demands to the legally necessary minimum. Devise workflows accordingly.

- Apply logic and don't ask networks to submit information that you already have and that is still valid.

- Always bear in mind that smooth and creative academic cooperation are the objective, not administrative overload.
- Constant dialogue: set up a system of stakeholder meetings to be able to quickly adapt to new developments.

- ... Encourage the enthusiasm of cooperation! Promote excellence!

One of the main tasks of the Central CEEPUS Office as the overall coordinating facility has been to harmonize different local procedures and obligations in such a way that a general system of implementation could be promoted, thus helping to assure that CEEPUS was operated the same way in all member states and showed "uniform appearance" as the Joint Committee of Ministers had postulated.

In order to achieve this objective, regular meetings of the National CEEPUS Offices, academic experts and the Senior Officials responsible for the program have been held to discuss current developments and accommodate new activities networks wanted to implement.

Each academic year, all submitted and formally correct network applications are evaluated by experts in each participating country using the "CEEPUS Scoring System" jointly developed and adapted at the Evaluation/ Senior Officials' Meetings.

To further help along user friendliness for networks, mobility applicants, experts and the CEEPUS administration, a comprehensive software solution covering all aspects of program implementation has been developed by the Central CEEPUS Office already at the very beginning of CEEPUS operations and continuously updated and enlarged ever since.

To promote excellence, the "Ministers' Prize of Excellence" was devised in 2002, to be awarded to outstanding networks each year:

To be eligible, a network has to have used at least 85 $\%$ of the overall total of scholarship months awarded to its participating units the previous academic year. Then the CEEPUS Scoring System is applied and the winner established. Having received the prize, a network has to observe a waiting period of three academic years before being eligible again.

\subsection{The proof of the pudding...}

But of course, the proof of the pudding is in the eating. Evidently CEEPUS met existing demands as per 1994/95 already 18 networks could be selected. Numbers have kept growing ever since. The application 2020/21 has yielded a record number of 118 network applications with almost 2000 HEI units ("participating units") involved. 
The average number of partners in a network has risen to fourteen, a large increase compared to the minimum requirement of three partners.

The number of applications that can be accepted - 85 for 2020/21 - is of course limited by the number of scholarship months available. For the selection round 2020/21 that meant that another 30 networks qualified to be put on the Waiting List. Only three network applications had to be rejected.

Once it was clear that networks could indeed perform well, demands were raised. Thus in 2003 the objective of Joint Degrees was introduced. Networks either operating or developing a Joint Program of any type where to be awarded more points during the selection procedure. By now the vast majority of CEEPUS networks are meeting this requirement.

\subsection{Branching out}

The CEEPUS Agreement being open for succession, nine new member states have acceded to the program so far. Croatia, the Czech Republic and Romania early on during CEEPUS I, Serbia and Montenegro (then still one country) have joined CEEPUS in 2004, Albania and (North) Macedonia in 2005 and Bosnia and Herzegovina in 2007. Moldova has acceded to CEEPUS in 2013.

The "CEEPUS Family" as stake holders on all levels like to refer to themselves, has managed to successfully enlarge networks and integrate the newcomers. Cooperation options with the industry or with institutions not eligible for CEEPUS have also been arranged for. These can participate as "Silent Partners", i.e. no CEEPUS grants can be received and a "Silent Partner" cannot coordinate a whole network. (CEEPUS Work Porgramme 2019-2021, Action 1, para 1.) Current examples for "Silent Partners" are scientific organizations such as the International GeoGebra Institute, the Sinus Society, SEFI (European Society for Engineering Education, Loughborough University) and companies, e.g. the Automotive Proving Ground Zala Ltd to name but a few.

\subsection{The CEEPUS incubator}

That's what networks like to call CEEPUS. Cooperation with other programs have always been encouraged by awarding extra points in the selection procedure. Again, networks have met the challenge, using CEEPUS as their "incubator" to test new schemes of cooperation in a user friendly and flexible setting. Once successful, the scheme is combined with other programs. Programs combined with CEEPUS are e.g. Erasmus +, Horizon 2020, COST, MSCA Bilateral Cooperations, EUSDR.

\subsection{The CEEPUS experience}

What a journey! Now, 25 years later, already in the second cycle of CEEPUS III, it can be said that CEEPUS has more than met the founders' hope to get their universities "Erasmus ready". CEEPUS networks have proven to be over-achievers, delivering much more than just smooth exchange actions. CEEPUS networks have delivered an impressive number of scientific papers and by focusing on $\mathrm{PhD}$ students are training the future scientific elite of the region - and the fact that they are regional networks with many destinations makes for brain circulation rather than brain drain. A follow up study on the last overall evaluation (Scheck \& Manahl, 2015) has shown that CEEPUS has become an important regional career factor, documenting career paths starting as a CEEPUS exchange student and then using CEEPUS contacts to successfully promote one's career, even ending up as head of a department (Scheck \& Manahl, 2016, p. 12).

\subsection{Case in point}

HU-28, Active Methods in Teaching and Learning Mathematics and Informatics, one of the biggest long serving and most successful CEEPUS networks, is an impressive case in point:

Started in 2001 as cooperation of seven partners from five countries, HU-28 now comprises 72 participating units from all CEEPUS countries. To attract so many new partners is a clear indication that remarkably interesting activities have been going on that new partners were glad to join. HU-28 also incorporates ten Silent Partners, namely important regional and international scientific societies. HU-28 uses all forms of CEEPUS activity and exchanges undergraduates, $\mathrm{PhD}$ students, young teaching staff as well as experienced university professors.

Several Joint Programs have been developed, ranging from M.Sc. programs in teaching informatics and mathematics to joint thesis supervision by thèse co-tutelle cooperations in $\mathrm{PhD}$ research programs. Recently network partners have developed a large new cooperation lead by the Didactical PhD program in Linz at the JKU STEM Education Centre (Johannes Kepler University STEM Education Centre.) 
Teaching materials have been jointly developed, making use of the helpful fact that partners understand each other's language, thus minimizing the need to use English as a lingua franca.

An on-line volume with ISBN number outlining activities of the CEEPUS summer university organized by HU-28 from July $6-17,2020$, can be viewed here: https://www.uni-miskolc.hu/ matsefi/Summer_ University_2020/

Last but not least, HU-28 also annually produces about thirty publications and research papers.

The driving force of this remarkable success story is the enthusiasm and engagement of highly skilled and dedicated academic staff who aim at teaching math and informatics the very best and engaging way. Sophisticated management skills are of the essence, of course.

\section{Conclusion}

What about a CEEPUS IV? After a quarter of a century of successful operation and having delivered on its original task, CEEPUS has no unique selling proposition concerning regional exchange actions any more. By now, most of its member states are Erasmus+ Program Countries. Albania, Bosnia - Hercegovina, Moldova and Montenegro are Erasmus+ Partner Countries. Other programs such as MSCA and COST are also open to CEEPUS countries. However, CEEPUS is still high in demand (Schuch, 2019, pp. 20-23).

CEEPUS has also morphed from a student exchange program into a teacher and $\mathrm{PhD}$ student exchange program. Since 2015/16 CEEPUS teacher and PhD mobilities have outnumbered student mobilities. As of 2017/18 this trend is even more pronounced.

What CEEPUS has, however, is a highly competent, dedicated and very enthusiastic set of well-connected regional researchers and teachers. CEEPUS is a brand standing for excellence and successful regional cooperation.

Defining new unique tasks that only CEEPUS can manage exceptionally well is key in developing a new attractive program generation. Some ideas for the "CEEPUS IV incubator":

- Given the current situation of having to cope with keeping up teaching during a pandemic, attractive online teaching models that can later blend well with "real time" lessons ("blended learning") are dearly needed. Even without a pandemic, such new teaching models are of great interest in view of rising numbers of students who are holding down jobs while studying.

CEEPUS networks such as HU-28 seem predestined to meet this challenge.

- The Joint Committee of Ministers could define "priority topic clusters" and provide political support in implementing solutions developed by such thematic network clusters, e.g. in dealing with regional environmental and climate change issues or autonomous driving. In both cases relevant networks are already operative.

- As for a new bold take on administration, in case a CEEPUS IV will indeed be focused on PhD students, closer formal cooperation between universities, research institutions and - where applicable partners from the industry might be worth considering. Applicants might then receive a "Regional Ticket" allowing for as many or as little exchange actions as desired, as long as basic conditions im terms of workload or achievements are met. The "Regional Ticket" might also cover online lessons and virtual practica.

- The basic legal framework also needs to be evaluated: Should CEEPUS IV again be based on an international agreement and if so, could there be several tracks defined in the Work Program and countries be allowed to opt for all tracks or only a some of them in a given period of time? Or should another set up be considered? If so, who should be the signatories? Would a new legal framework really be an advantage?

Too bold? This cannot work? Why not? As CEEPUS has shown so far, bold ideas can work very well if tackled the right way. There is a strong basis of valuable and experienced networks that would be up to the job. Of course, a lot of homework by the member states would be needed in order to arrange for the proposals listed but where there is a will there might also be a CEEPUS IV.

\section{References}

CEEPUS III Agreement (2010). Deposit of the Central CEEPUS Office in Vienna. https://www.ceepus.info/files/c3/gi/C3Agreement. pdf

CEEPUS III Work Programme 2019-2021. https://www.ceepus.info/ files/c3/gi/C3WorkProgram.pdf

CEEPUS Scoring System: https://www.ceepus.info/files/ceepus3/ WhatlsNewAppRound.pdf p. $20 \mathrm{ff}$. 
Scheck E., Manahl C. (2015): Evaluation CEEPUS III: Frequent travellers under the microscope. [ZSI - Centre for Social Innovations.] DOI:10.22163/fteval.2015.6

Scheck E., Manahl C. (2016): Evaluation CEEPUS III: Frequent travellers under the microscope. A Supplement to the Evaluation of CEEPUS' teacher mobility. [ZSI - Centre for Social Innovations. Internal material of the Central CEEPUS Office.]

Schuch K. (2019): The Uptake of European Programmes in the CEEPUS Cooperation Area. [ZSI - Centre for Social Innovations.] https://www.zsi.at/de/object/project/5259 